\title{
Efeito do SBS Epoxidado como um Agente Compatibilizante para Obtenção de Nanocompósitos de SBS/Argila Cloisite 10A
}

\author{
Patrícia Alves da Silva, Marly Antonia Maldaner Jacobi, Kelly Silva dos Santos, \\ Ricardo Vinicius Bof de Oliveira, Raquel Santos Mauler \\ Universidade Federal do Rio Grande do Sul - UFRGS \\ Ronilson Vasconcelos Barbosa \\ Universidade Federal do Paraná - UFPR
}

\begin{abstract}
Resumo: Atualmente, cargas nanométricas são utilizadas para desenvolver novos materiais poliméricos com melhor desempenho mecânico. Neste trabalho, foi empregada uma mistura de borracha termoplástica de poli(estireno-b-butadieno-b-estireno) (SBS) e de argila Montmorilonita (Cloisite 10A) via intercalação por fusão. Uma suspensão de Cloisite 10A em ciclohexano foi adicionada a SBS em solução. Para maior compatibilidade entre a argila e a borracha termoplástica utilizou-se uma borracha termoplástica epoxidada como agente compatibilizante. Neste estudo, a proporção argila compatibilizante foi de 0,6 até 3,0 para verificar a influência desta proporção nas propriedades mecânicas dos nanocompósitos. As propriedades morfológicas e mecânicas dos nanocompósitos foram avaliadas através da difração de raios X, microscopia eletrônica de transmissão, ensaio de tração, análise dinâmico-mecânica (DMA) e avaliação do torque final da mistura. A combinação de argila/conpatibilizante favoreceu a formação de estruturas mais alongadas de argila que promoveram o ganho de módulo e tensão com pequena redução de alongamento.
\end{abstract}

Palavras-chave: Nanocompósitos, borracha termoplástica do tipo SBS, borracha epoxidada.

\section{Epoxidized SBS as Compatibilizer for Obtaining SBS/Clay Cloisite 10A Nanocomposites}

Abstract: Nanoscale fillers have been used to develop new polymeric materials with better mechanical performance. In this study, a mixture of thermoplastic rubber, poly (styrene-b-butadiene-b-styrene) (SBS), and Montmorillonite (Cloisite 10A) was obtained via the melt intercalation. A suspension of Cloisite 10A in cyclohexane was added to SBS in solution. To increase the compatibility between the clay and the thermoplastic rubber an epoxidized rubber was used as coupling agent. The proportion of clay and compatibilizer was changed from 0.6 to 3.0 to probe the influence of this ratio on the mechanical properties of the nanocomposites. The morphological and mechanical properties were evaluated using X-ray diffraction, transmission electron microscopy, tensile testing, dynamic mechanical analysis (DMA) and evaluation of the final torque of the mixture. The combination of clay /compatibilizer favored the formation of elongated structures, leading to an increased modulus and strength with a small reduction in elongation.

Keywords: Nanocomposites, thermoplastic rubber, epoxidized rubber.

\section{Introdução}

Uma das vantagens do uso de nanocompósitos em relação aos compósitos convencionais é o ganho de propriedades com um pequeno acréscimo de $\operatorname{carga}^{[1]}$. A produção de nanocompósitos pode ser realizada basicamente de três formas: através de polimerização in situ, em solução e no estado fundido ou melt blending ${ }^{[2]}$. As vantagens de formar nanocompósitos por intercalação no estado fundido são que o processamento não utiliza solventes orgânicos e o menor custo envolvido devido à sua compatibilidade com os processos de transformação de termoplásticos ${ }^{[3-6]}$.

Inicialmente, as principais pesquisas envolvendo nanocompósitos foram dedicadas principalmente para os termoplásticos e resinas termorrígidas ${ }^{[7,8]}$. No entanto a área de elastômeros apresenta uma gama promissora de aplicações.
Existem algumas pesquisas nesta área de nanocompósitos de látex ${ }^{[9]}, \mathrm{SBR}^{[10]}$, borracha natural ${ }^{[11]}$, borracha termoplástica do tipo $\mathrm{SBS}^{[12]}$, nanocompósitos híbridos ${ }^{[13]}$ e blendas de nanocompósitos de borracha com $\mathrm{PP}^{[14]}$. Geralmente a adição da argila na matriz polimérica favorece no ganho de propriedades mecânica. Liao e colaboradores ${ }^{[2]}$ formaram um nanocompósito de SBS através da dispersão de 2,5 phr de argila em SBS utilizando tolueno, seguido de precipitação em etanol onde o nanocompósito apresentou estrutura intercalada com ganho de $48 \%$ no módulo de armazenamento e em elongação. Alguns autores utilizaram diferentes tipos de compatibilizantes de acordo com a matriz polimérica para aumentar a interação matriz/carga. Entre os principais compatibilizantes destacam-se o anidrido maleico grafitizado (MA) em diferentes 
polímeros: SEBS-g-MA ${ }^{[15]}$, PP-g-MA ${ }^{[16]}$, EPR-g-MA ${ }^{[17]}$, EPDM-g-MA ${ }^{[18]}$; o ácido acrílico (AA), PP-g-AA ${ }^{[19]}$ e o metacrilato de metila (MMA) com polímeros estirênicos $^{[20]}$. Arroyo ${ }^{[11]}$ utilizou borracha epoxidada como compatibilizante e obteve uma morfologia mais homogênea favorecendo no ganho de propriedades físicas e mecânicas dos nanocompósitos. Assim, um estudo mais detalhado a respeito do efeito dos grupos polares deste compatibilizante no processo de esfoliação da argila na obtenção de nanocompósitos de SBS é crucial para correlacionar a morfologia obtida com as propriedades finais desses materiais. O objetivo deste trabalho foi avaliar o efeito de diferentes proporções de uma borracha epoxídica na dispersão da MMT na matriz de SBS e, consequentemente, em suas propriedades dinâmico-mecânicas e mecânicas.

\section{Experimental}

\section{Materiais}

Os materiais utilizados na preparação dos nanocompósitos foram: a borracha termoplástica do tipo SBS fornecida pela Petroflex com $30 \% \mathrm{~g} / \mathrm{g}$ de estireno, a argila montmorilonita organofílica Cloisite 10A modificada por um sal de amônio quaternário, contendo os grupos dimetil, benzil e ácido graxo hidrogenado e fornecido pela Rockwood Additives, uma borracha epoxidada com grau de epoxidação de $13,9 \mathrm{~mol} \%$ fornecida pelo laboratório de nossa universidade como um agente compatibilizante ${ }^{[21]}$ e o solvente ciclohexano da Nuclear.

\section{Preparação dos nanocompósitos e caracterização}

Os nanocompósitos de SBS foram preparados conforme patente depositada PI0701345-0 $0^{[22]}$. As misturas foram preparadas em câmara de mistura Haake a $120{ }^{\circ} \mathrm{C}$ por 5 minutos com velocidade de $60 \mathrm{rpm}$. A formulação dos nanocompósitos foi estudada conforme a Tabela 1 .

Placas de $100 \mathrm{~mm} \times 120 \mathrm{~mm} \times 2,0 \mathrm{~mm}$ foram moldadas por compressão em prensa hidráulica Carver à temperatura $130{ }^{\circ} \mathrm{C}$ com pré-aquecimento de 5 minutos e pressão de 4,5 Lbf por 5 minutos. As análises de raios $\mathrm{X}$ foram realizadas com fonte de cobre $(\lambda=1,54 \mathrm{~nm}$ com ângulo inicial de $1^{\circ}$ e final de $45^{\circ}$ com incremento de $\left.0,5^{\circ}\right)$. Os ensaios de tração foram realizados na máquina universal de ensaios Emic com célula de carga de $500 \mathrm{~N}$. Os corpos de prova foram confeccionados de acordo com a norma ASTM D 412 tipo C. As análises dinâmico-mecânicas (DMA) foram realizadas em um aparelho TA Instruments modelo QA 800 operando em modo "single cantillever" com dimensões aproximadas de $10,00 \times 60,00 \times 2,00 \mathrm{~mm}^{3}$. As medidas foram realizadas a $1 \mathrm{~Hz}$. As amostras foram analisadas em perfil de temperaturas de -100 até $130{ }^{\circ} \mathrm{C}$ com taxa de $3{ }^{\circ} \mathrm{C}$ $\min ^{-1}$. Para obtenção dos cortes dos nanocompósitos foi utilizada câmara criogênica acoplada ao ultramicrótomo LMC CXR e esta foi mantida na temperatura de $-80^{\circ} \mathrm{C}$. Posteriormente, com auxílio de lâmina de diamante, obteve-se cortes ultrafinos que foram analisados em um microscópio eletrônico de transmissão JEOL JEM-1200 Ex II com tensão de aceleração de $80 \mathrm{kV}$.

\section{Resultados e Discussão}

\section{Morfologia e propriedades mecânicas dos nanocompósitos de SBS/C10A: a influência da concentração de um agente compatibilizante}

Segundo Ray e seus colaboradores, existem três principais morfologias na obtenção dos nanocompósitos ${ }^{[23]}$, intercalada, intercalada-floculada e esfoliada. $\mathrm{Na}$ morfologia intercalada-floculada o polímero penetra entre as camadas da argila, mantendo ainda sua estrutura (organização). Na Figura 1 é possível observar que a argila $10 \mathrm{~A}$ tem um pico característico (d 001) em $2 \theta=4,58^{\circ}$, que corresponde a uma distância interplanar de $\mathrm{d}=1,93 \mathrm{~nm}$. Os nanocompósitos de SBS apresentaram uma parcial intercalação referente aos picos em $2 \theta=4,24^{\circ}$ e $2,07^{\circ}$, mostrando um aumento de $1,93 \mathrm{~nm}$ para $2,10 \mathrm{~nm}$ e de $1,93 \mathrm{~nm}$ para $4,28 \mathrm{~nm}$, respectivamente, na distância interplanar da MMT. Estes resultados sugerem que os nanocompósitos de SBS apresentaram uma distribuição heterogênea de suas

Tabela 1. Formulação dos nanocompósitos de SBS.

\begin{tabular}{ccc}
\hline Amostra (\%) \\
(SBS,MMT,SBSepox)
\end{tabular}

$\mathrm{P}=$ Proporção (MMT/SBSepox). 
cadeias entre as folhas de MMT independente do uso de um compatibilizante. O nanocompósito com a proporção $\mathrm{P}=3,0$ apresentou uma diminuição em sua intensidade possivelmente caracterizando uma estrutura parcialmente esfoliada $^{[6]}$. O pico em $6,2^{\circ}$ com $\mathrm{d}=1,45 \mathrm{~nm}$ corresponde ao pico característico da argila sódica, mostrando que uma pequena porcentagem da argila pode não ter modificado totalmente pelo sal de amônio quartenário ${ }^{[24]}$. Outra sugestão é que durante o processamento, as argilas poderiam ter perdido o sal, mas para esta afirmação, outras análises e estudos deveriam ser realizados.

Muitas vezes a técnica de raios X torna-se inconclusiva quanto à morfologia do nanocompósito. Os difratogramas fornecem um indicativo de intercalação-esfoliação, porém a presença de estruturas aglomeradas e/ou agregadas pode estar mascarada. Assim, a microscopia eletrônica de transmissão (MET) permite conhecer com mais detalhamento o tipo de morfologia ou mistura de morfologias que um nanocompósito pode apresentar ${ }^{[16]}$.

As Figuras 2 e 3 apresentam as imagens de MET dos nanocompósitos de SBS. Na Figura 2a e b $\mathrm{P}=0$ amostras sem compatibilizante a argila obteve morfologia aglomerada. Em (c) e (d) $\mathrm{P}=0,6$ com $5 \%$ de compatibilizante a argila apresentou orientação devido a presença do compatibilizante. Na Figura $3 \mathrm{a}$ e b $\mathrm{P}=1,0$ foi observada estrutura intercalada e alongada. Em (c) e (d) $\mathrm{P}=2,0$ também foi observado partes aglomeradas

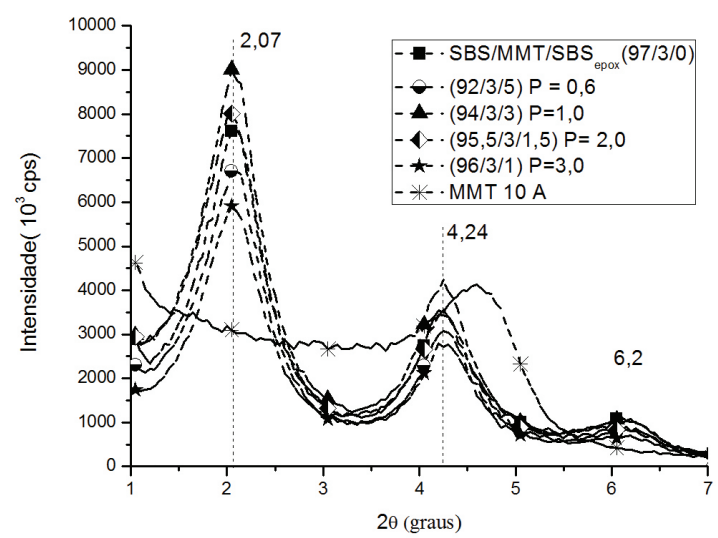

Figura 1. Difratograma de raios $\mathrm{X}$ dos nanocompósitos de SBS/ MMT $10 \mathrm{~A}$. e intercaladas. Em (e) e (f), $\mathrm{P}=3,0$ amostras com menor percentual de compatibilizante foi observada estrutura orientada e alongada. Nas Figuras 2 e 3 foi possível observar que a presença da borracha epoxidada como agente compatibilizante orientou as folhas de argila produzindo estruturas mais alongadas o que pode contribuir para o ganho de propriedades mecânicas ${ }^{[25]}$.

As Figuras 4 e 5 apresentam as curvas de tensão $\mathrm{x}$ deformação dos nanocompósitos de SBS. A Tabela 2 apresenta os valores obtidos dos ensaios de tração. A reação de epoxidação permite a introdução de grupos polares nos polidienos melhorando suas propriedades finais ${ }^{[26,27]}$, além de aumentar as interações entre os grupos polares da MMT e a matriz de SBS. A introdução da borracha epoxidada na borracha SBS aumentou os valores de tensão em relação à borracha pura (Tabela 2), reduziu seus valores de alongamento devido à introdução dos grupos epoxídicos polares, mantendo sua rigidez. Porém, quanto menor a quantidade de compatibilizante, maiores foram os valores de tensão obtidos. A mistura que continha $1 \%$ de compatibilizante $(\mathrm{P}=3,0)$ foi a mistura que obteve o maior valor de tensão.

$\mathrm{Na}$ Tabela 2 e Figuras 4 e 5 foi possível observar um aumento de módulo e tensão em todas as proporções de argila/compatibilizante e, também, houve uma pequena redução no alongamento da SBS. A adição de argila reduziu mais o alongamento comparado com a presença do compatibilizante devido à interação argila/SBS. Este aumento de propriedades deve-se a maior interação entre a carga e a matriz polimérica ${ }^{[28]}$. A combinação argila/compatibilizante apresentou um comportamento de reforço rígido, aumentando a tensão e módulo com redução do alongamento. A redução no alongamento dos nanocompósitos pode ser atribuída pela maior orientação da nanocarga na matriz polimérica preferencialmente na direção do ensaio de tração, confirmado pelas imagens de MET. Este comportamento foi alcançado quando a proporção argila/compatibilizante foi aumentada, visto que o sinergismo entre compatibilizante/argila produziu estruturas mais alongadas e orientadas.

A proporção $\mathrm{P}=3$ apresentou as melhores características morfológicas (maior esfoliação e ou orientação da argila) e mecânicas. Isto se deve ao fato de que na proporção $3: 1$, menores quantidades de grupos epóxidicos poderiam estar ajudando na dispersão da

Tabela 2. Propriedades mecânicas dos nanocompósitos de SBS com diferentes proporções de argila/compatibilizante.

\begin{tabular}{|c|c|c|c|c|}
\hline $\begin{array}{c}\text { Amostra (\%) } \\
\left(\mathrm{SBS} / \mathrm{MMT} / \mathrm{SBS}_{\mathrm{epox}}\right) \\
\end{array}$ & $\begin{array}{c}\text { Tensão máx } \\
\text { (MPa) }\end{array}$ & $\begin{array}{c}\text { Alongamento na ruptura } \\
(\%)\end{array}$ & $\begin{array}{c}\text { Módulo Tangente (5\%) } \\
\text { (MPa) }\end{array}$ & $\begin{array}{c}\text { Torque Final } \\
(\mathbf{N m}) \\
\end{array}$ \\
\hline$(100 / 0 / 0)$ & $10,9 \pm 1,4$ & $1328 \pm 86$ & $11,3 \pm 0,8$ & 15,7 \\
\hline$(95 / 0 / 5)$ & $11,7 \pm 1,2$ & $1225 \pm 19$ & $12,1 \pm 0,4$ & 15,5 \\
\hline$(97 / 3 / 0)$ & $12,9 \pm 1,0$ & $1111 \pm 11$ & $15,4 \pm 1,2$ & 15,4 \\
\hline$(92 / 3 / 5) \mathrm{P}=0,6$ & $11,3 \pm 0,1$ & $1146 \pm 28$ & $17,1 \pm 0,2$ & 14,4 \\
\hline$(97 / 0 / 3)$ & $12,4 \pm 0,3$ & $1173 \pm 18$ & $13,0 \pm 2,0$ & 15,3 \\
\hline$(94 / 3 / 3) \mathrm{P}=1,0$ & $10,2 \pm 0,3$ & $1067 \pm 63$ & $21,2 \pm 4,3$ & 14,8 \\
\hline$(98,5 / 0 / 1,5)$ & $13,1 \pm 0,9$ & $1245 \pm 9,8$ & $10,5 \pm 0,9$ & 15,4 \\
\hline$(95,5 / 3 / 1,5) \mathrm{P}=2,0$ & $11,5 \pm 0,1$ & $1072 \pm 17$ & $16,0 \pm 1,5$ & 14,6 \\
\hline$(99 / 0 / 1)$ & $13,9 \pm 0,7$ & $1264 \pm 12$ & $9,6 \pm 1,1$ & 15,4 \\
\hline$(96 / 3 / 1) \mathrm{P}=3,0$ & $13,1 \pm 0,3$ & $1082 \pm 42$ & $19,0 \pm 1,5$ & 14,5 \\
\hline
\end{tabular}

$\mathrm{P}=$ proporção argila/compatibilizante. 


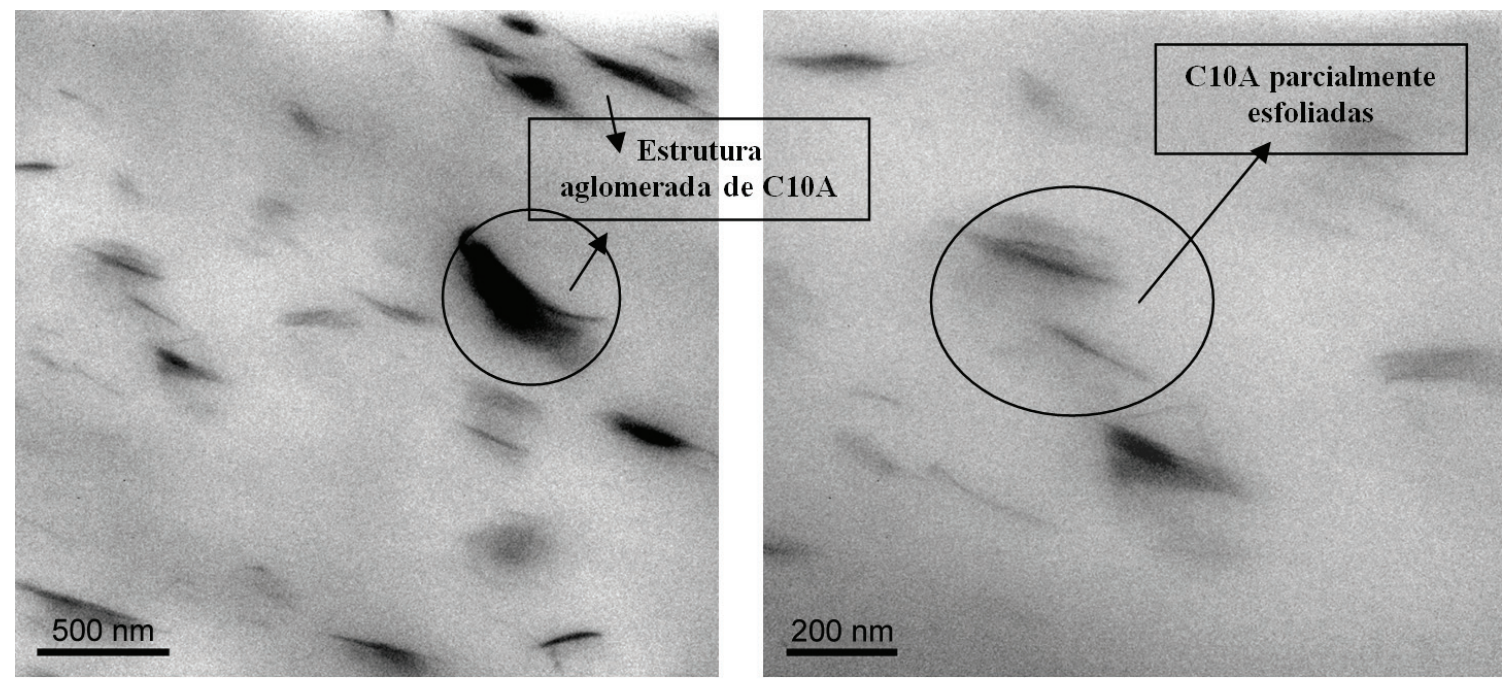

(a) $(97,3,0) P=0$ sem compatibilizante

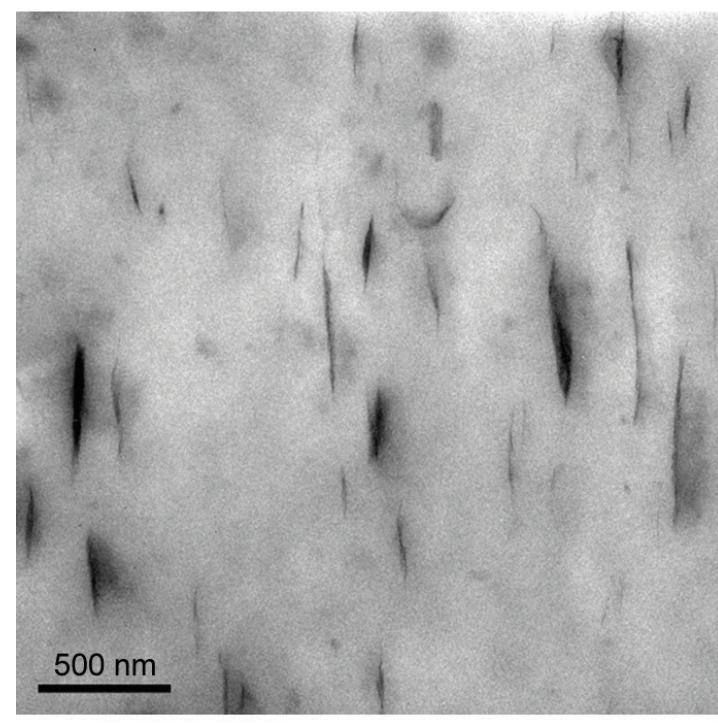

(c) $(92,3,5) \quad P=0,6$ (b) $(97,3,0) \quad P=0$ sem compatibilizante

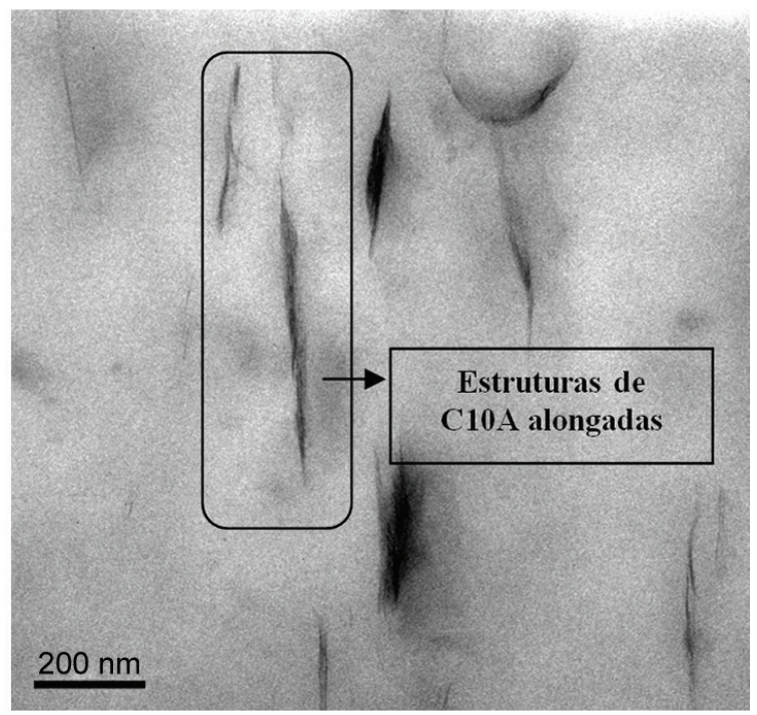

(d) $(92,3,5) \quad P=0,6$

Figura 2. Imagens de MET dos nanocompósitos de SBS/MMT/SBS ${ }_{\text {epox }}$, em diferentes proporções (P) argila/compatibilizante: (A) e (B), $\mathrm{P}=0$; (C) e (D) $\mathrm{P}=0,6$ em diferentes aumentos, respectivamente, nos tamanhos de $500 \mathrm{~nm}$ e $200 \mathrm{~nm}$.

argila e mantendo as propriedades mecânicas da SBS com ganho de módulo. Esta proporção também apresentou menor número de estruturas aglomeradas que as demais proporções e uma morfologia parcialmente esfoliada com estruturas alongadas no que auxiliou no ganho de propriedades mecânicas.

Além disso, os nanocompósitos com $\mathrm{SBS}_{\text {epox }}$ apresentaram menores valores de torque comparados com os obtidos com a borracha epoxidada e a borracha pura. A obtenção deste resultado sugere maior interação da SBS com argila e o compatibilizante.

As Figuras 6 e 7 apresentam o módulo de armazenamento $\left(E^{\prime}\right)$ para a borracha pura e para as misturas com compatibilizante sem argila e com argila. Nas curvas de DMA dos nanocompósitos de SBS pode ser observado que os valores de $E^{\prime}$ diminuem à medida que a temperatura aumenta, mostrando um patamar borrachoso em temperaturas da ordem de -50 a $100{ }^{\circ} \mathrm{C}$. Foi observado que para todos os percentuais de $\mathrm{SBS}_{\text {epox }}$ o valor de módulo de armazenamento foi inferior ao da borracha pura, exceto a mistura contendo $3 \%$ que apresentou maiores valores de módulo. O percentual de $5 \%$ de compatibilizante apresentou menor valor de módulo de armazenamento. Estes resultados sugerem que o percentual de $3 \%$ de compatibilizante seria o valor ótimo de mistura e $5 \%$ poderia ser um valor em excesso, onde para estes valores as propriedades são inferiores a amostra pura.

Na Figura 7 foi observado que a combinação de argila com o compatibilizante promoveu um ligeiro aumento na rigidez da SBS indicando uma boa interação entre a argila/compatibizante e matriz polimérica. 


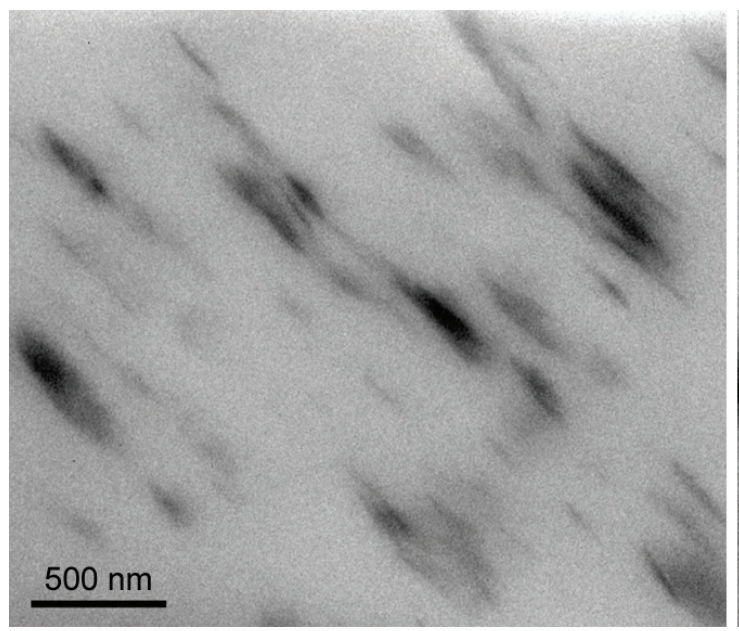

(a) $(94,3,3) P=1,0$

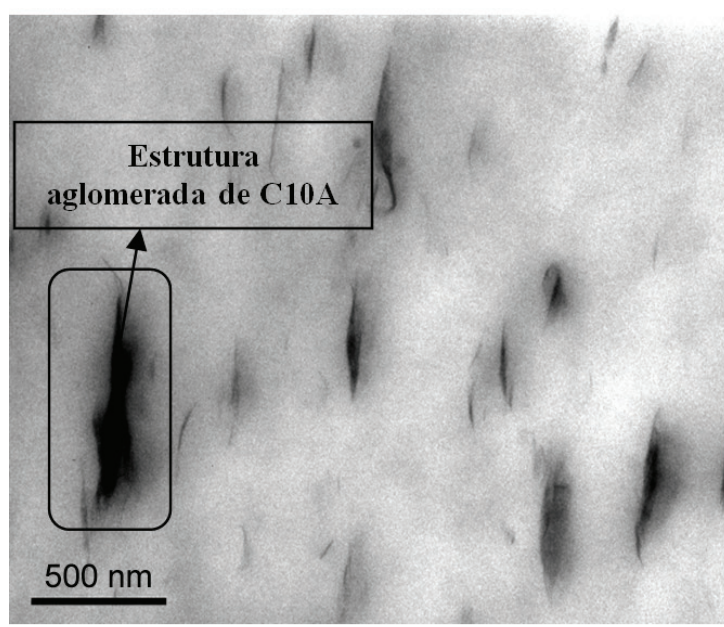

(c) $(95.5,3,1.5) \quad P=2,0$

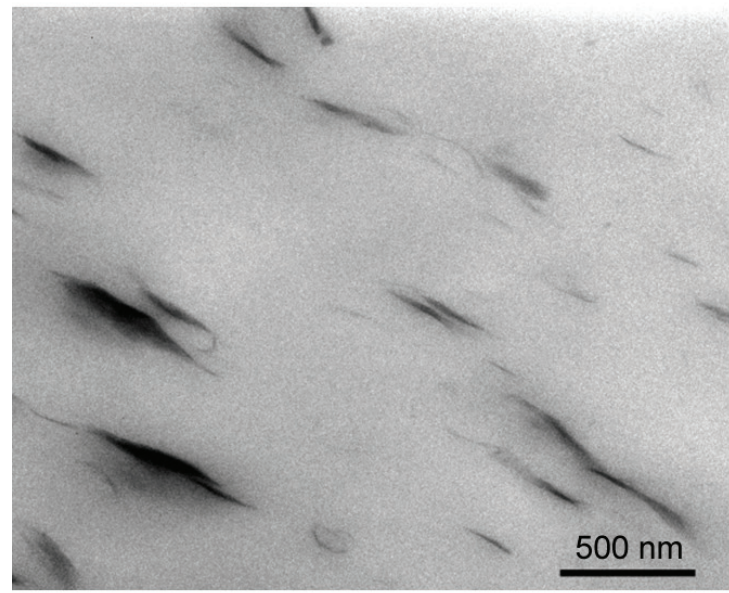

(e) $(96,3,1) \quad P=3,0$

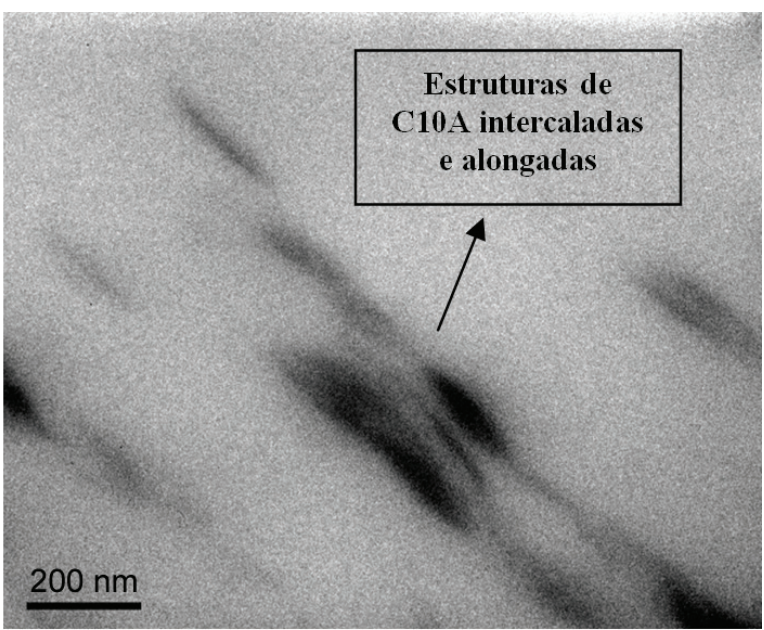

(b) $(94,3,3) P=1,0$

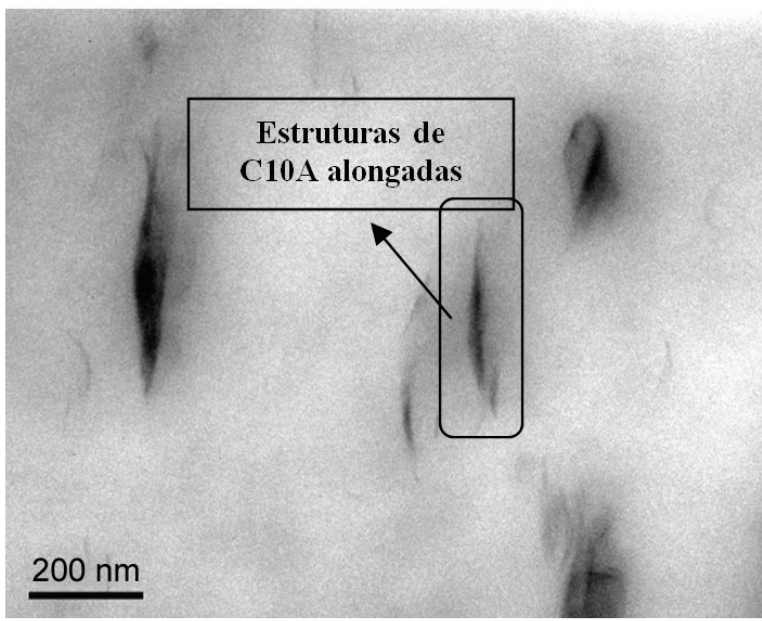

(d) $(95.5,3,1.5) \quad P=2,0$

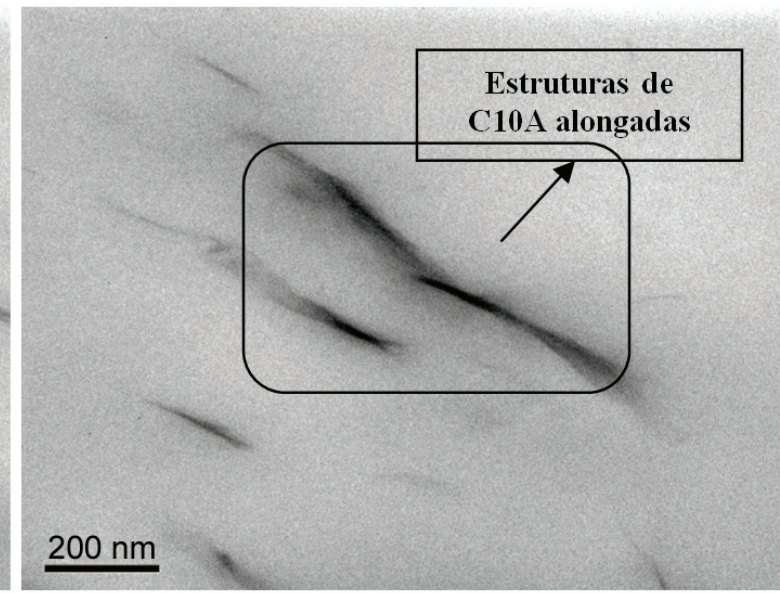

(f) $(96,3,1) P=3,0$

Figura 3. Imagens de MET dos nanocompósitos de SBS/MMT/SBS ${ }_{\text {epox }}$, em diferentes proporções (P) argila/compatibilizante: (A) e (B) $\mathrm{P}=1,0 ;(\mathrm{C})$ e (D) $\mathrm{P}=2,0 ;$ (E) e (F) $\mathrm{P}=3,0$ em diferentes aumentos, respectivamente, nos tamanhos de $500 \mathrm{~nm}$ e $200 \mathrm{~nm}$. 


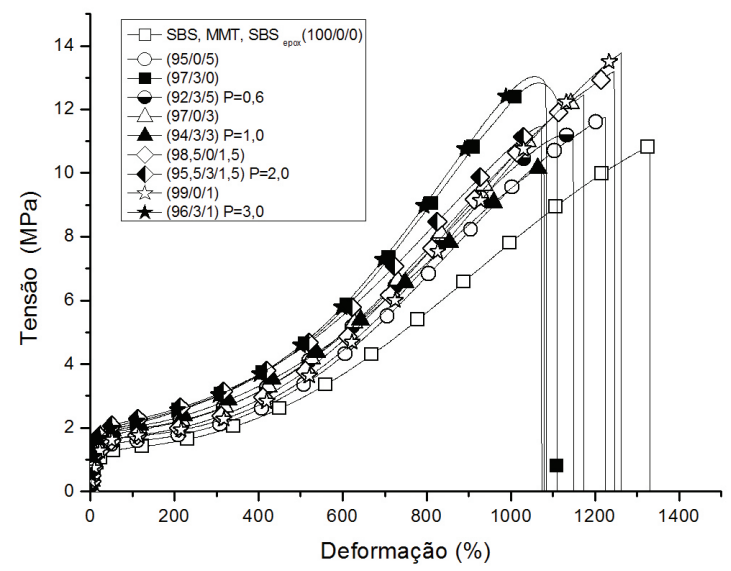

Figura 4. Curvas tensão-deformação para o SBS puro, SBS e $\mathrm{SBS}_{\text {epox }}$ e seus nanocompósitos em diferentes proporções de argila/compatibilizante.

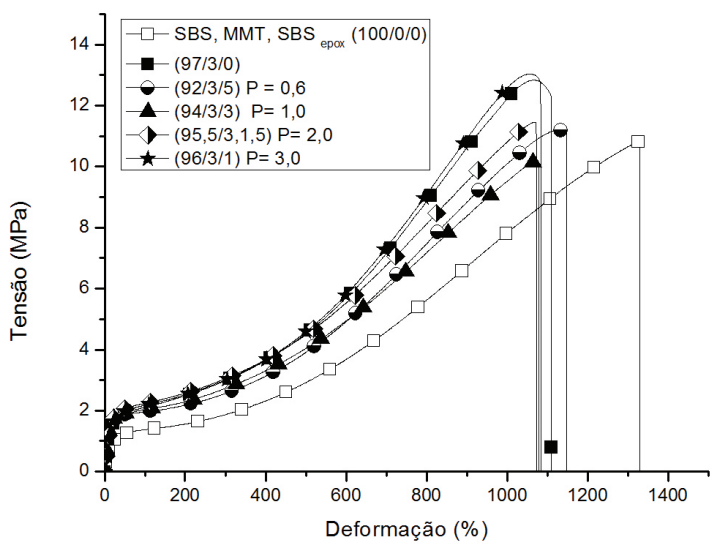

Figura 5. Curvas tensão-deformação para o SBS puro e seus nanocompósitos em diferentes proporções de argila/ compatibilizante.

Na temperatura acima da temperatura ambiente todos os nanocompósitos apresentam rigidez maior que a borracha pura evidenciando as interações entre o compatibilizante e a carga. O nanocompósito $(94,3,3)$ apresentou menor módulo de armazenamento e o com maior quantidade de compatibilizante $(92,3,5)$ apresentou o maior. Nas misturas com somente o compatibilizante este comportamento foi o contrário.

A Tabela 3 apresenta os valores de temperatura de transição vítrea das amostras na fase butadiênica, através da análise por DMA. Na SBS pura o valor da $\mathrm{T}_{\mathrm{g}}$ foi de $-68,67{ }^{\circ} \mathrm{C}$ e a amostra $(95,5 / 3 / 1,5)$ apresentou $\mathrm{T}_{\mathrm{g}}^{\mathrm{g}}$ de $-74,40{ }^{\circ} \mathrm{C}$, para as demais amostras também ocorreu um pequeno deslocamento da $\mathrm{T}_{\mathrm{g}}$ para temperatura mais baixas. O deslocamento da temperatura de transição vítrea para menores valores de temperatura, indica maior flexibilidade entre as cadeias das misturas em relação à amostra pura.

$\mathrm{Na}$ Figura 8 a amostra contendo $5 \%$ de compatibilizante apresentou maior amortecimento. Uma maior quantidade de compatibilizante, por ser preferencialmente miscível com a fase do butadieno, aumentou a sua fração volumétrica, que é caracterizado

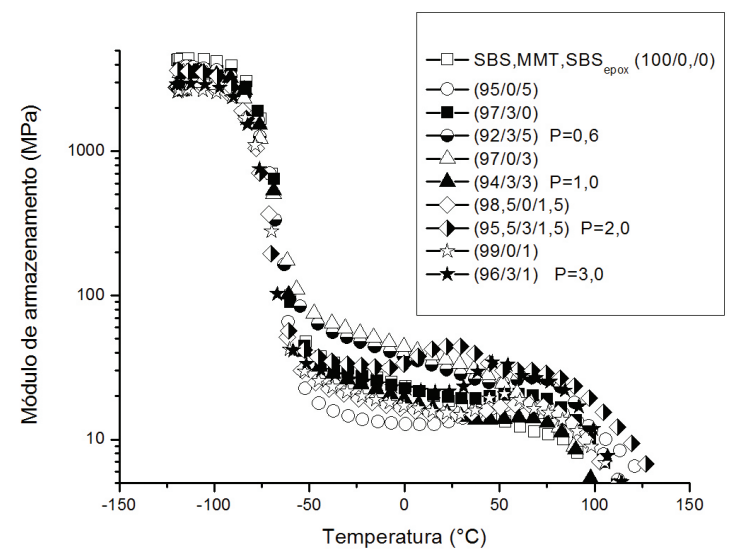

Figura 6. Curvas de DMA para as misturas: SBS pura, SBS/ SBS $_{\text {epox }}$ e SBS/MMT/SBS ${ }_{\text {epox}}$.

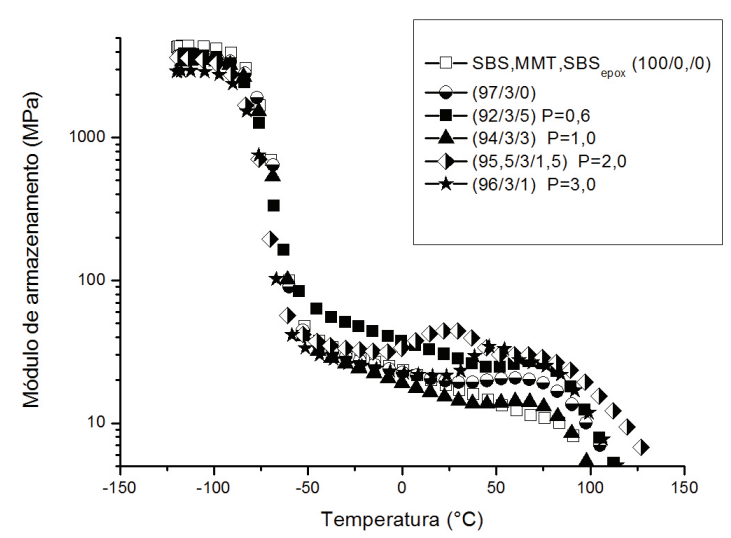

Figura 7. Curvas de DMA para as misturas: SBS/MMT/SBS ${ }_{\text {epox }}$

Tabela 3. Temperatura de transição vítrea (Tg) da SBS pura, ${\mathrm{SBS} / \mathrm{SBS}_{\mathrm{epox}} \text { e SBS/MMT/SBS }}_{\mathrm{ep}}$

\begin{tabular}{cc}
\hline $\begin{array}{c}\text { Amostra }(\%) \\
\left(\mathbf{S B S} / \mathbf{M M T} / \mathbf{S B S}_{\text {epox }}\right)\end{array}$ & $\begin{array}{c}\mathbf{T g} \\
\left({ }^{\circ} \mathbf{C}\right)\end{array}$ \\
\hline$(100 / 0 / 0)$ & $-68,67$ \\
$(95 / 0 / 5)$ & $-68,72$ \\
$(97 / 3 / 0)$ & $-68,87$ \\
$(92 / 3 / 5) \mathrm{P}=0,6$ & $-70,14$ \\
$(97 / 0 / 3)$ & $-68,98$ \\
$(94 / 3 / 3) \mathrm{P}=1,0$ & $-68,61$ \\
$(98,5 / 0 / 1,5)$ & $-71,45$ \\
$(95,5 / 3 / 1,5) \mathrm{P}=2,0$ & $-74,40$ \\
$(99 / 0 / 1)$ & $-72,39$ \\
$(96 / 3 / 1) \mathrm{P}=3,0$ & $-71,40$ \\
\hline
\end{tabular}

$\mathrm{P}=$ proporção argila/compatibilizante.

pelo aumento de amortecimento. Contudo, a presença da argila provocou um aumento da rigidez da cadeia caracterizado pela redução do amortecimento. A adição de argila e compatibilizante na SBS pura, teve a tendência de uma pequena redução do valor da $\mathrm{T}_{\mathrm{g}}$, efeitos competitivos da fase rígida com a fase flexível e efeito carga-matrix $^{[28,29]}$.

Por meio da Figura 9 foi possível observar que o pico característico do estireno (aproximadamente $80{ }^{\circ} \mathrm{C}$ ) foi 


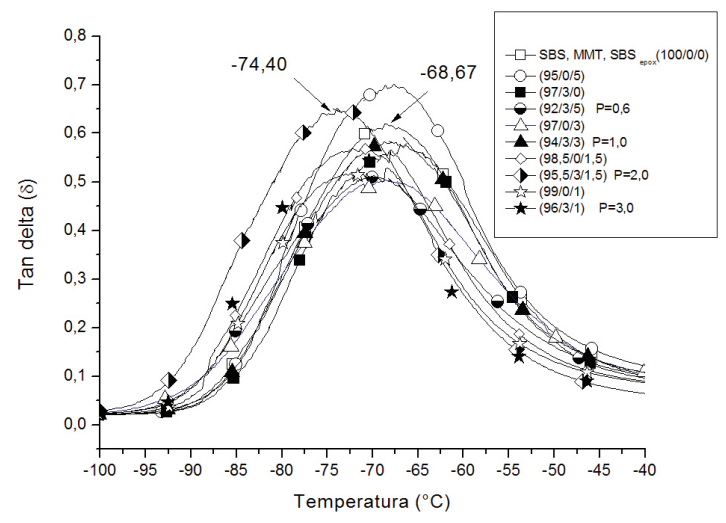

Figura 8. Curvas de Tan delta para as misturas: (A) SBS/SBS e (B) SBS/MMT/SBS ${ }_{\text {epox }}$ - fase butadiênica.

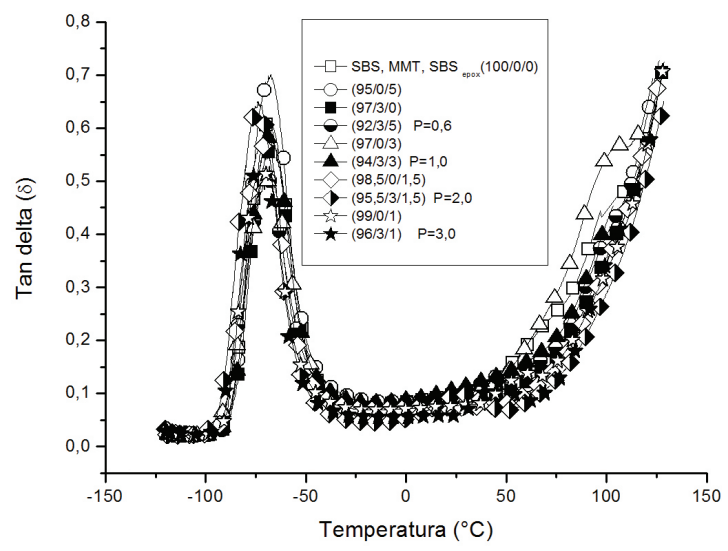

Figura 9. Curvas de Tan delta para as misturas: (A) SBS/SBS e (B) SBS/MMT/SBS

deslocado para temperaturas mais baixas para a amostra pura e para a amostra contendo $3 \%$ de compatibilizante. A presença de compatibilizante juntamente com a argila afeta as fases, tornando a fase estirênica mais flexível e a fase butadiênica com menor amortecimento.

\section{Conclusões}

Os ensaios mecânicos mostraram que a adição da argila aumentou o módulo e a borracha epoxidada ajudou a aumentar a tensão da borracha. Todas as proporções apresentaram bons resultados, destacando-se a proporção argila/compatibilizante $3: 1$, que foi a proporção com menor quantidade de compatibilizante e mais favorável no ganho de propriedades finais, pois houve melhor sinergia entre a mistura argila/compatibilizante e a matriz de SBS. Os grupos epoxídicos presentes no compatibilizante juntamente com a argila, tornaram a borracha SBS mais rígida na fase butadiênica e mais flexível na fase estirênica, visto que este compatibilizante promoveu uma boa interação entre os componentes. A presença do compatibilizante orientou a argila e promoveu a formação de estruturas mais alongadas favorecendo um ganho de propriedades mecânicas. A adição da borracha epoxidada como compatibilizante e a avaliação de diferentes proporções argila/compatibilizante favoreceram no ganho de propriedades e na escolha da melhor proporção de argila/compatibilizante.

\section{Agradecimentos}

Os autores agradecem ao CNPq, PRONEX/ FAPERGS pelo apoio financeiro.

\section{Referências Bibliográficas}

1. Chakraborty, S.; Kar, S.; Dasgupta, S.; Mukhopadhyay, R.; Bandyopadhyay, S.; Joshi, M. \& Ameta, S. C. - Polym. Test., 29, p.181 (2010). http://dx.doi.org/10.1016/j. polymertesting.2009.10.008

2. Liao, M.; Zhu, J.; Xu, H.; Li, Y. \& Shan, W. - J. Appl. Polym. Sci., 92, p.3430 (2004). http://dx.doi.org/10.1002/ app. 20372

3. Hong, C. K.; Kim, M. J.; Oh, S. H.; Lee, Y. S. \& Nah, C. - J. Ind. Eng. Chem., 14, p.236 (2008). http://dx.doi. org/10.1016/j.jiec.2007.11.001

4. Souza, M. A.; Pessan, L. A. \& Rodolfo Júnior, A. - Polímeros, 16, p. 257 (2006). http://dx.doi.org/10.1590/ S0104-14282006000400003

5. Lai, S. M. \& Chen, C. M. - Eur. Polym. J., 43, p.2254 (2007). http://dx.doi.org/10.1016/j.eurpolymj.2007.03.014

6. Morelli, F. C. \& Ruvolo Filho, A. - Polímeros, 20, p.121 (2010). http://dx.doi.org/10.1590/ S0104-14282010005000014

7. Alexandre, M. \& Dubois, P. - Mater. Sci. Eng., 28, p.1 (2000). http://dx.doi.org/10.1016/S0927-796X(00)00012-7

8. Jia, Q.; Zheng, M.; Chen, H. \& Shen, R. - Mater. Lett., 60, p.1306 (2006). http://dx.doi.org/10.1016/j. matlet.2005.11.018

9. Varghese, S.; Kocsis, K. - Polymer, 44, p.4921 (2003). http://dx.doi.org/10.1016/S0032-3861(03)00480-4

10. Zhang, Z.; Zhang, L.; Li, Y. \& Xu, H. - Polymer, 46, p.129 (2005). http://dx.doi.org/10.1016/j.polymer.2004.11.008

11. Arroyo, M.; López-Manchado, M. A, Valentin, J. L. \& Carretero, J. - Compos. Sci. Tecnol., 67, p.1330 (2007) http://dx.doi.org/10.1016/j.compscitech.2006.09.019

12. Xu, H.; Li Y. \& Yu, D. - J. Appl. Polym. Sci., 98, p.146 (2005). http://dx.doi.org/10.1002/app.22029

13. Mariano, R. M.; Nunes, R. C. R.; Visconte, L. L. Y.; Altstaedt, V. - Polímeros, 23, p.123 (2013).

14. Silva, P. A.; Jacobi, M. M.; Schneider. L. K.; Barbosa, R. V.; Coutinho, P. A.; Oliveira, R. V. B. \& Mauler, R. S. - Polym. Bull., 64, p.245 (2010). http://dx.doi.org/10.1007/ s00289-009-0159-3

15. Farahani, R. D. \& Ramazani, A. S. A. - Mat. Des., 29, p.105 (2008).

16. Chrissopoulou, K. \& Anastasiadis, S. H. - Eur. Polym. J., 47, p.600 (2011). http://dx.doi.org/10.1016/j. eurpolymj.2010.09.028

17. Chow, W. S.; Bakar, A. A.; Ishak, M.; Karger-Kocsis, J. \& Ishiaku, U. S - Eur. Polym. J., 41, p.687 (2005). http:// dx.doi.org/10.1016/j.eurpolymj.2004.10.041

18. Pasbakhsh, P.; Ismail, H.; Fauzi, M. N. A. \& Bakar, A. A. - Polym. Test., 28, p.548 (2009). http://dx.doi. org/10.1016/j.polymertesting.2009.04.004 
19. Chinellato, A. C.; Vidotti, S. E.; Hu, G.-H. \& Pessan, L. A. - Compos. Sci. Tecnol., 70, p.458 (2010). http://dx.doi. org/10.1016/j.compscitech.2009.11.020

20. Keskkula, H.; Paul, D. R.; McCreedy, M. \& Henton, D. E. - Polymer, 28, p.2063 (1987). http://dx.doi.org/10.101 6/0032-3861(87)90042-5

21. Jacobi, M. M.; Santin, C. K. \& Schuster, R. H. V. - Kautsch Gummi Kunstst, 57, p.82 (2004).

22. Silva, P. A.; Coutinho, P. L. A.; Campos, C. R. A.; Jacobi, M. A. M.; Cassinelli, J. R. D.; Schneider, L. K. A.; Lira, C. H.; Barbosa, R. V. \& Mauler, R. S. - "Processo para obtenção de um material compósito em que uma nanocarga é aplicada a um polímero e material compósito resultante", Instituto nacional de propriedade industrial, Rio de Janeiro (2007).

23. Ray, S. S. \& Okamoto, M. - Prog. Polym. Sci., 28, p.1539 (2003).
24. Madaleno, L.; Thomsen, J. S. \& Pinto, J. C. - Compos. Sci. Tecnol., 70, p.804 (2010). http://dx.doi.org/10.1016/j. compscitech.2010.01.016

25. Balakrishnan, S.; Start, P. R.; Raghavan, D. \& Hudson, S. D. - Polymer, 46, p.11255 (2005). http://dx.doi. org/10.1016/j.polymer.2005.10.053

26. Rocha, T. L. A.; Jacobi, M. M. \& Schuster, R. H. - Polímeros, 16, p.111 (2006).

27. Nakason, C.; Wannavilai, P. \& Kaesaman, A. - J. Appl. Polym. Sci., 101, p.3046 (2006). http://dx.doi.org/10.1002/ app. 23926

28. Thomas, S. P.; Thomas, S.; Bandyopadhyay, S.; Wurm, A.; Schick, C. - Compos. Sci. Tecnol., 68, p.3220 (2008).

29. Rooj, S.; Das, A. \& Heinrich, G. - Eur. Polym. J., 47, p. 1746 (2011). http://dx.doi.org/10.1016/j.eurpolymj.2011.06.007

Enviado: 03/01/13 Reenviado: 07/05/13 Aceito: 10/06/13 